OPEN ACCESS

Edited by:

Helmut Fickenscher,

University of Kiel, Germany

Reviewed by:

Egidia Miftode,

Grigore T. Popa University of Medicine and Pharmacy, Romania

Makoto Kurano,

The University of Tokyo, Japan

*Correspondence:

Xingtao Zhou

doctzhouxingtao@163.com

Wenjun Cao

wgkjyk@aliyun.com

Specialty section:

This article was submitted to

Viral Immunology,

a section of the journal

Frontiers in Immunology

Received: 15 April 2021

Accepted: 19 July 2021

Published: 06 August 2021

Citation:

Li S, Qiu Y, Tang L, Wang Z,

Cao W, Zhou $X$ and Sun $X$ (2021)

Association of Ocular Surface

Diseases With SARS-CoV-2

Infection in Six Districts of China:

An Observational Cohort Study.

Front. Immunol. 12:695428.

doi: 10.3389/fimmu.2021.695428

\section{Association of Ocular Surface Diseases With SARS-CoV-2 Infection in Six Districts of China: An Observational Cohort Study}

\author{
Shengjie $L^{1,2}{ }^{1,}$ Yichao Qiu ${ }^{1}$, Li Tang ${ }^{1}$, Zhujian Wang ${ }^{1}$, Wenjun Cao ${ }^{1,2,3,4 *}$, \\ Xingtao Zhou ${ }^{2,3,4,5,6 *}$ and Xinghuai Sun ${ }^{2,3,4}$ \\ ${ }^{1}$ Clinical Laboratory, Eye \& ENT Hospital, Fudan University, Shanghai, China, ${ }^{2}$ Eye Institute and Department of Ophthalmology, \\ Eye \& ENT Hospital, Fudan University, Shanghai, China, ${ }^{3}$ NHC Key Laboratory of Myopia (Fudan University), Key Laboratory of \\ Myopia, Chinese Academy of Medical Sciences, Shanghai, China, ${ }^{4}$ Shanghai Key Laboratory of Visual Impairment and \\ Restoration, Shanghai, China, ${ }^{5}$ Shanghai Research Center of Ophthalmology and Optometry, Shanghai, China, 6 Shanghai \\ Engineering Research Center of Laser and Autostereoscopic 3D for Vision Care (20DZ2255000), Shanghai, China
}

The severe acute respiratory syndrome coronavirus 2 (SARS-CoV-2) viruses is mainly transmitted through respiratory droplets. Notably, some coronavirus disease 2019 (COVID-19) patients have ocular manifestations, including conjunctival hyperaemia, chemosis, epiphora, and increased secretions. However, the association between SARS-CoV-2 and ocular surface diseases is poorly described. Between May 2020 and March 2021, a total of 2, 0157 participants from six districts of China were enrolled. Serum samples were tested for immunoglobulin $G$ and $M$ ( $\lg G$ and $\lg M$ ) antibodies against the SARS-CoV-2 spike protein and nucleoprotein using magnetic chemiluminescence enzyme immunoassays. Throat swabs were tested for SARS-CoV-2 RNA using RT-PCR assays in a designated virology laboratory. Fisher exact, $\chi^{2}$ test, and logistic regression analysis were performed. Of 2, 0157 serum samples tested, 1, 755 (8.71\%) were from ocular surface diseases, 1, 2550 (62.26\%) from no-ocular surface diseases (ocular diseases except ocular surface diseases), 5, 852 (29.03\%) from no-ocular diseases. SARS-CoV-2 prevalence for the combined measure was $0.90 \%$ (182/2, 0157). Seroprevalence of SARS-CoV-2 was significantly $(\mathrm{p}<0.05)$ higher in the population with ocular surface diseases $(2.28 \%, 40 / 1755)$ compared with no-ocular surface diseases $(0.70 \%, 88 / 1$, $2550)$, and no-ocular diseases $(0.92 \%, 54 / 5,852)$. Similar results were also observed with respect to sex, age, time, and districts. Logistic regression analyses revealed that ocular surface diseases [ocular surface diseases vs. no-ocular diseases $(\mathrm{p}=0.001, \mathrm{OR}=1.467$, 95\% $\mathrm{Cl}=1.174-1.834)$; ocular surface diseases vs. no-ocular surface diseases $(p<0.001$, $\mathrm{OR}=2.170,95 \% \mathrm{Cl}=1.434-3.284)]$ were associated with increased risk of susceptible to SARS-CoV-2 infection. In a word, there was a significant association between ocular surface disease and SARS-CoV-2 infection. Therefore, increasing awareness of eye protection during the pandemic is necessary, especially for individuals with ocular surface diseases.

Keywords: SARS- CoV-2, immunoglobulin G, immunoglobulin M, ocular surface diseases, cross-sectional study, seroprevalence, COVID - 19 


\section{INTRODUCTION}

Coronavirus disease 2019 (COVID-19), a novel respiratory disease caused by severe acute respiratory syndrome coronavirus 2 (SARSCoV-2), emerged in December 2019. As of July 13, 2021, 187,086,096 laboratory-confirmed cases of COVID-19 were reported, with more than 4,042,921 known fatalities (https:// covid19.who.int/). The SARS-CoV-2 virus is mainly transmitted through respiratory droplets and by close contact with infected individuals (1). Notably, some patients have ocular manifestations, including conjunctival hyperaemia, chemosis, epiphora, and increased secretions (2-4). Furthermore, a recent study reported that the SARS-CoV-2 receptor angiotensin-converting enzyme 2 (ACE2) and entry protease transmembrane serine protease 2 (TMPRSS2) are strongly detected in human ocular surfaces, indicating that they may provide SARS-CoV-2 with a portal of entry (5). Thus, ocular surface may as a possible site of virus entry and also as a source of contagious infection.

Little information is available about the prevalence of SARS$\mathrm{CoV}-2$ in population with ocular diseases, and the association between SARS-CoV-2 and ocular surface diseases is poorly described. To our knowledge, only one study performed a retrospective small sample $(\mathrm{n}=1100)$ survey found no association between ocular symptoms and COVID-19 positivity in an outpatient population (6). Therefore, estimating the seroprevalence of SARS-CoV-2 in patients with ocular surface diseases is crucial for controlling the transmission of SARS-CoV-2 through ocular surface.

Serological tests, such as validated assays for immunoglobulin G (IgG) and immunoglobulin M (IgM) antibodies against SARSCoV-2, have the advantages of easy serum sample collection and high throughput (7). Antibodies can be detected in most COVID-19 patients 7-14 days after diagnosis $(8,9)$ and remain at high levels four months after diagnosis (10). In this study, we assayed IgG and $\operatorname{IgM}$ antibodies to estimate the seropositivity rates to assess the association of ocular surface diseases with SARS-CoV-2 infection.

\section{METHODS}

\section{Study Design and Participants}

A total of 2,0157 individuals were enrolled in this study from May 2020 to March 2021: 6,233 from Shanghai, 3,946 from Jiangsu province, 3,014 from Anhui province, 3,004 from Zhejiang province, 1,787 from Jiangxi province, and 2,173 from Henan province (Figure 1 and Table 1). The subjects who have been vaccinated against SARS-CoV-2 were excluded from this study $(\mathrm{n}=81)$.

All subjects underwent medical examinations performed by respective specialty physicians. Meanwhile, all subjects underwent ophthalmic examination conducted by an ophthalmologist specialist. The ocular diseases were diagnosed by an ophthalmologist. The time frame of the ocular condition was also recorded. In the present study, the patients with ocular diseases were newly diagnosed between May 2020 to March 2021 when they came to the hospital, and the blood of the patients was collected and measured immediately.
The ocular surface diseases group included corneal diseases, conjunctival diseases, dry eye, eyelid disease, lacrimal apparatus diseases, etc. The no-ocular surface diseases group included ocular diseases except ocular surface diseases. The no-ocular diseases group included all the subjects except patients with ocular diseases.

\section{Ethics Approval}

The Medical Ethics Committees of Eye and ENT Hospital of Fudan University approved this study, and the study adhered to the principles of the Declaration of Helsinki. Informed consent was obtained from all participants. For individuals younger than 18 years, consent was provided by parents or a legal guardian.

\section{Laboratory Measurements}

Throat swab samples were collected from May 2020 through March 2021. All samples were immediately transported to the laboratory to maintain the cold chain and tested within 12 hours of collection. Throat swabs were tested for SARS-CoV-2 RNA using RT-PCR assays in a designated virology laboratory (Zhongke Runda, Shanghai, China).

Serum samples were also collected from May 2020 through March 2021. All samples were analysed within eight hours of collection. IgG and IgM antibodies against the SARS-CoV-2 spike protein and nucleoprotein were measured using a commercially available magnetic chemiluminescence enzyme immunoassay kit (Bioscience, Chongqing, China), per the manufacturer's instructions. Antibody levels were expressed as the ratio of the chemiluminescence signal to the cut-off $(\mathrm{S} / \mathrm{CO})$ value. An $\mathrm{S} / \mathrm{CO}$ value higher than 1 for either IgG or IgM was considered positive. If the $\mathrm{S} / \mathrm{CO}$ value was higher than 0.7 but lower than 1.2 , the serum sample was re-analysed to confirm the results. Serological assays for SARS-CoV-2 infection have been validated in a previous study (11), which as follow: serum of 447 patients with end-stage kidney disease that were collected as a negative control and serum of 242 patients with COVID-19 confirmed by the viral RT-PCR test as a positive control and calculated the sensitivity and specificity of the assay. The serological test showed specificity of $99.3 \%$ (444 of 447) and 100\% (447 of 447) for IgG and IgM antibodies, respectively. Moreover, interference test reported that rheumatoid factor $(1500 \mathrm{IU} / \mathrm{ml})$, nonspecific IgG antibody (38.8g/l), on-specific IgM antibody (4.8g/l), antinuclear antibodies, and anti-mitochondrial antibody didn't have the potential interference on the results.

Since the IgG and IgM levels should decrease as time passed from the infection. Thus, we set the S/CO value at 0.5 levels for either IgG or IgM which might be infected with COVID-19 in the past (Table S1).

\section{Statistical Analysis}

A sample size calculation was undertaken in order to determine the study's recruitment sample sizes. We used an open-source calculator to calculate the minimal required sample size based on the probability of a type I error of alpha at 5\% (two-sided), type II error of beta at $30 \%$, and the normal prevalence of COVID-19 among the population in China, which is approximately $1 \%$. 


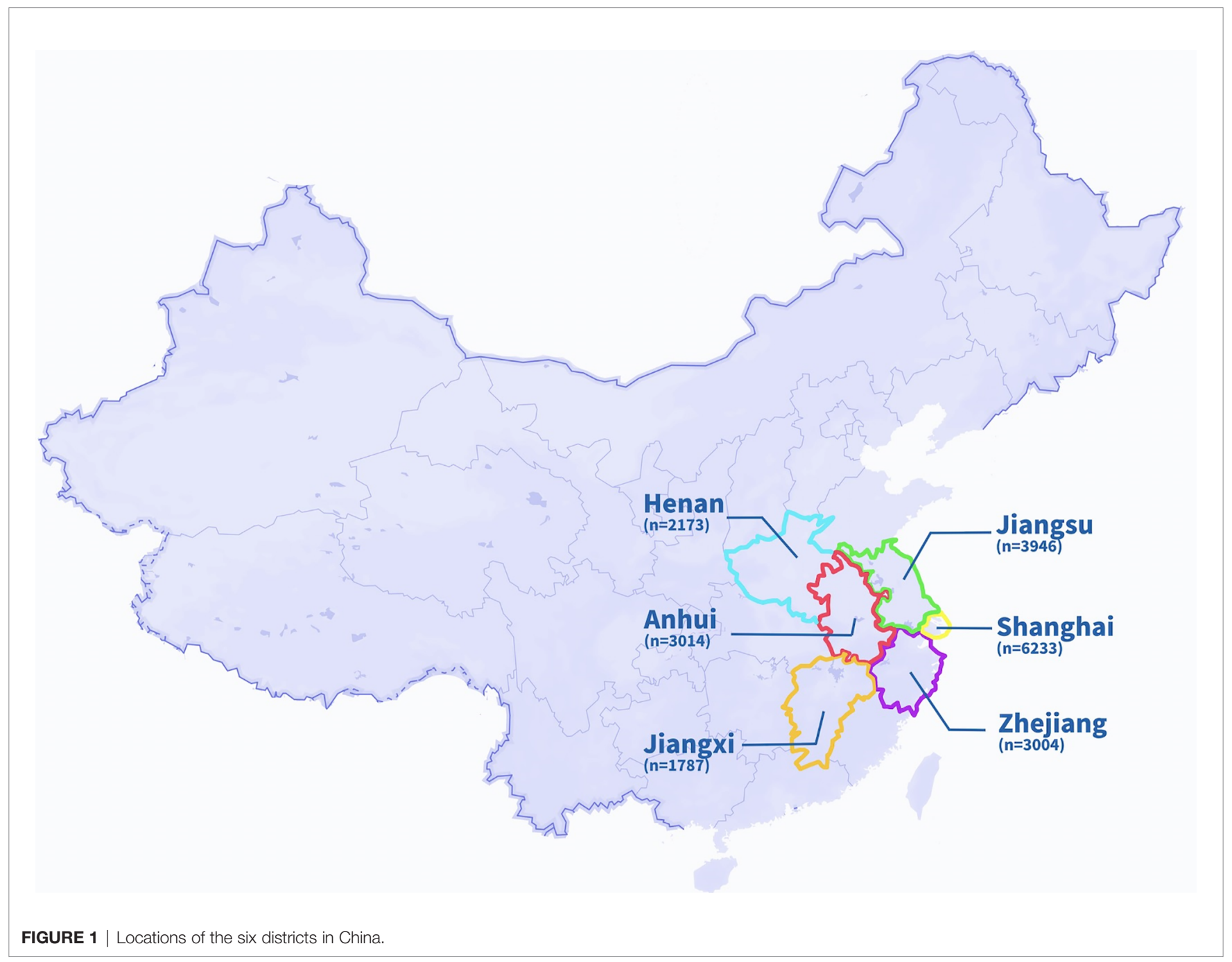

This calculation yielded a sample consisting of at least 5,293 participants. Categorical variables were presented as counts and percentages. Fisher exact and $\chi^{2}$ test was used for comparison between the groups. The results of logistic regression were presented as OR and corresponding 95\% confidence intervals (CIs). All analyses were performed using the Statistical Package for the Social Sciences software, version 13.0 (SPSS Inc., Chicago, IL, USA). Figures were created using GraphPad Prism 6
(GraphPad Software, La Jolla, CA, USA). A value of $\mathrm{P}<0.05$ was considered statistically significant.

\section{RESULTS}

Of all specimens, 1, 755 (8.71\%) were from ocular surface diseases, 1, 2550 (62.26\%) from no-ocular surface diseases, 5,

TABLE 1 | Number of clinical specimens for antibodies to SARS-CoV-2 in six districts.

\begin{tabular}{|c|c|c|c|c|c|c|}
\hline & Shanghai & Jiangsu & Anhui & Henan & Jiangxi & Zhejiang \\
\hline \multicolumn{7}{|l|}{ Sex } \\
\hline Male & 3021 & 1961 & 1538 & 1012 & 800 & 1361 \\
\hline Female & 3212 & 1985 & 1476 & 1161 & 987 & 1643 \\
\hline \multicolumn{7}{|l|}{ Age, y } \\
\hline $0-18$ & 356 & 279 & 219 & 123 & 85 & 236 \\
\hline $19-49$ & 2227 & 1913 & 1283 & 685 & 719 & 1361 \\
\hline $50-64$ & 1722 & 994 & 832 & 646 & 547 & 794 \\
\hline$\geq 65$ & 1928 & 760 & 680 & 719 & 436 & 613 \\
\hline Total & 6233 & 3946 & 3014 & 2173 & 1787 & 3004 \\
\hline
\end{tabular}


852 (29.03\%) from no-ocular diseases (Table 2). In this study, all the participants were RT-PCR negative for SARS-CoV-2 from throat swabs. The levels of IgM and IgG antibodies are presented in Figure 2. SARS-CoV-2 prevalence for the combined measure was $0.90 \%(182 / 2,0157)$. The seroprevalence in ocular surface diseases, no-ocular surface diseases (ocular diseases except ocular surface diseases), and no-ocular diseases was $2.28 \%$ (40/1755), $0.70 \%(88 / 12550)$, and $0.92 \%$ (54/5852), respectively. The seroprevalence of antibodies to SARS-CoV-2 was particularly high in the population with ocular surface diseases $(\mathrm{p}<0.05)$ (Table 2). Furthermore, the seroprevalence of $\operatorname{IgM}^{+}(1.42 \%$, $0.39 \%, 0.53 \%)$ and $\operatorname{IgG}^{+}(1.25 \%, 0.43 \%, 0.68 \%)$ was also particularly high in the population with ocular surface diseases $(\mathrm{p}<0.05)$ compared with no-ocular surface diseases and noocular diseases, respectively (Table 2). Owing to the ocular surface diseases are composed with various diseases, we further analysis the seroprevalence of antibodies to SARS-CoV-2 among different types of ocular surface diseases (Table 3).

We further set the S/CO value at 0.5 levels to see whether similar results were also observed. The seroprevalence in ocular surface diseases, no-ocular surface diseases, and no-ocular diseases was 3.48\% (61/1755), $1.95 \%$ (245/12550), and $1.98 \%$ (116/5852), respectively. The seroprevalence of antibodies to
SARS-CoV-2 was also particularly high in the population with ocular surface diseases, similar results were also observed in male and female subgroups (Table S1).

Based on sex, the subjects were categorized into male and female subgroup. The seroprevalence of SARS-CoV-2 was also particularly higher in ocular surface diseases compared with noocular surface diseases and no-ocular diseases in male $(2.00 \%$, $0.54 \%, 073 \%)$ and female $(2.46 \%, 0.86 \%, 1.10 \%)$ subgroup ( both $<0.05$ ) (Table 2 and Figure 3). Similar results were also observed when SARS-CoV-2 $\mathrm{IgM}^{+}$and $\mathrm{IgG}^{+}$prevalence was compared among ocular surface diseases, no-ocular surface diseases and no-ocular diseases groups with respect to sex, respectively ( $\mathrm{p}$ both $<0.05$ ) (Table 2 ).

Furthermore, in each district, SARS-CoV-2 prevalence was higher in ocular surface diseases compared with others $(\mathrm{p}<0.05)$ (Table 2). A similar result was observed when SARS-CoV-2 prevalence was compared among ocular surface diseases, noocular surface diseases and no-ocular diseases groups with respect to sex (Table 2 and Figure 4). Similar results were also observed when SARS-CoV-2 $\mathrm{IgM}^{+}$and $\mathrm{IgG}^{+}$prevalence was compared among ocular surface diseases, no-ocular surface diseases and no-ocular diseases groups with respect to district and sex, respectively (Figure 4).

TABLE 2 | SARS-CoV-2 seroprevalence by sex and districts.

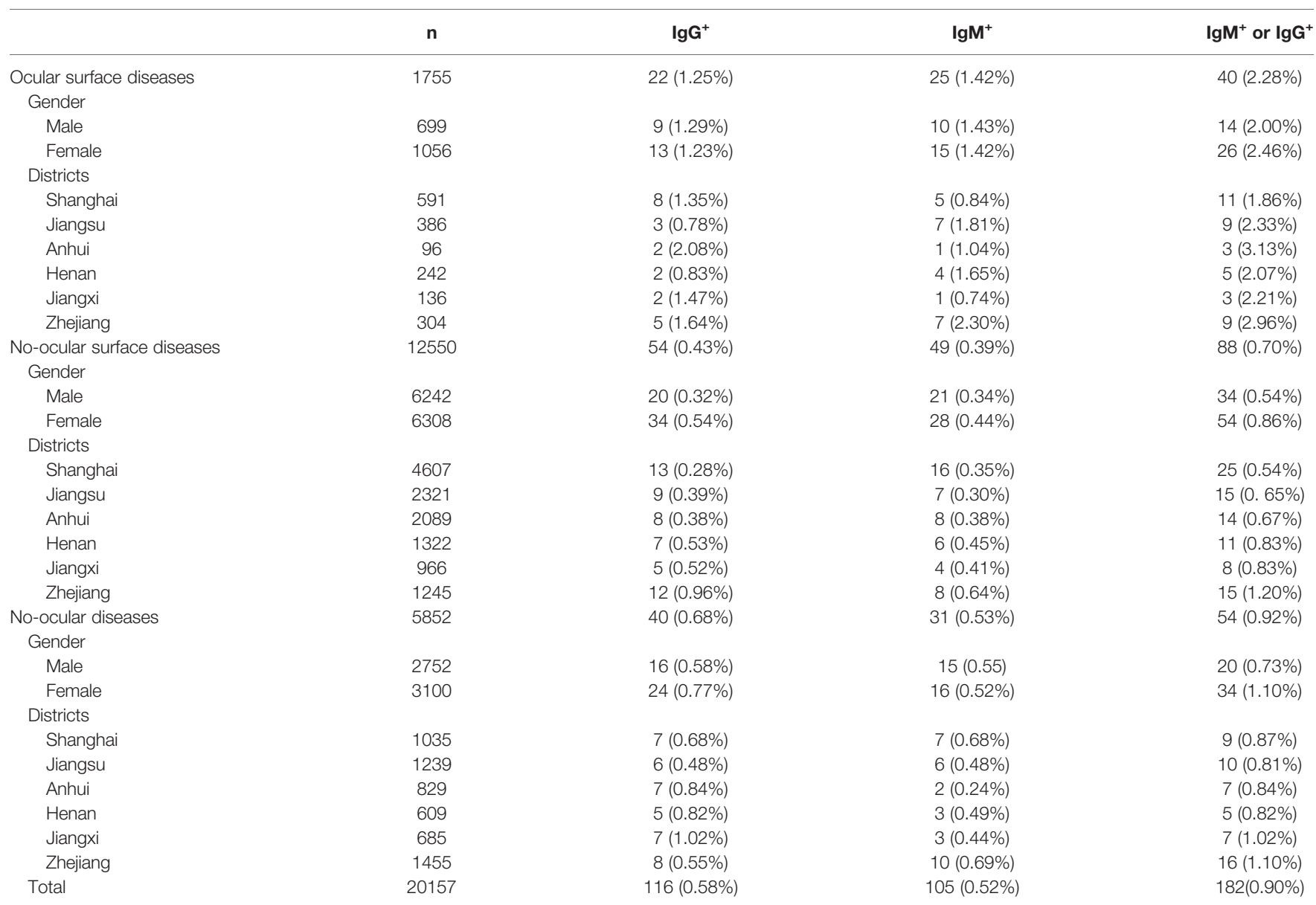




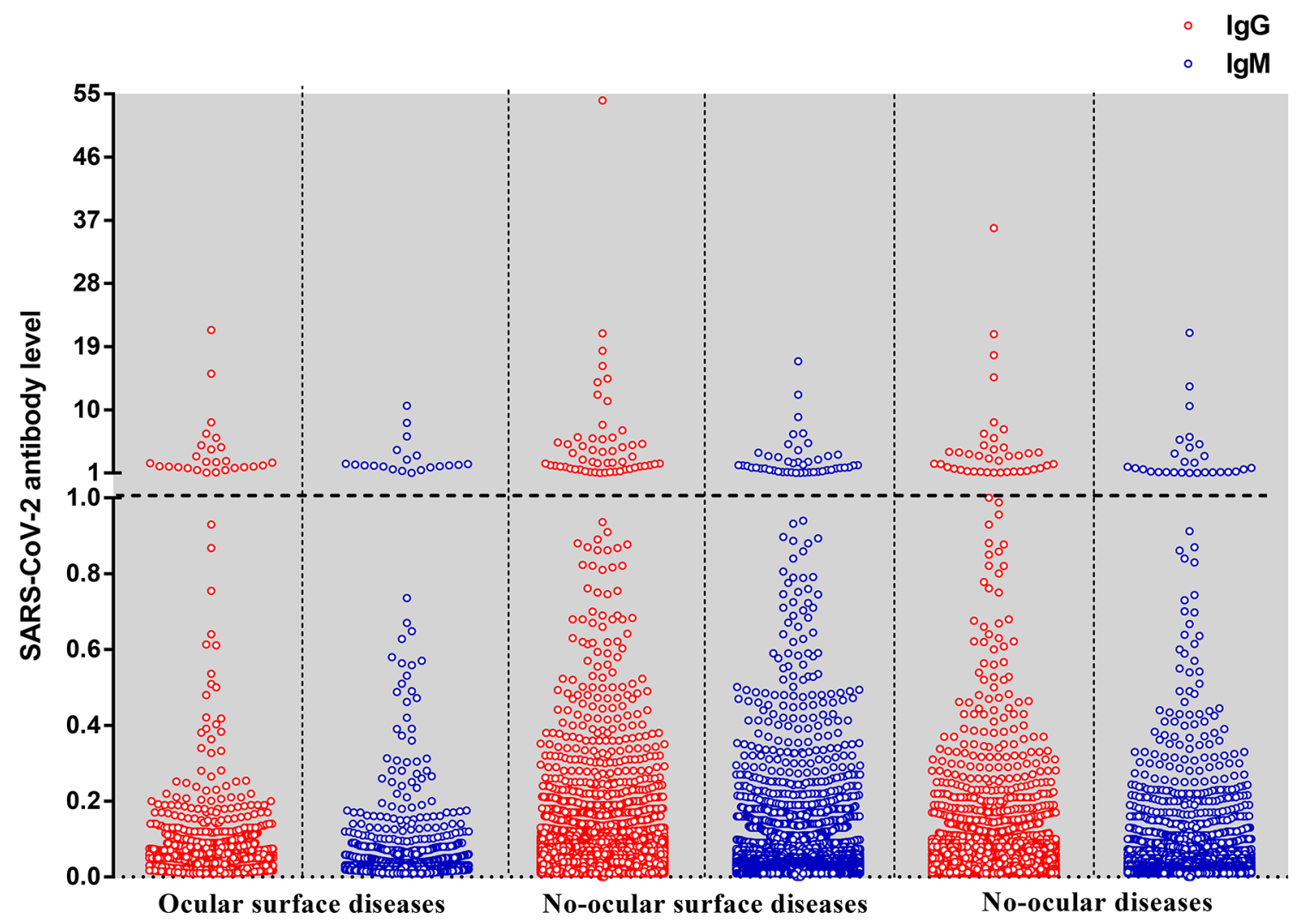

FIGURE 2 | The level of IgG and IgM antibodies against the nucleoprotein and a peptide from the spike protein of SARS-CoV-2 by the type of diseases. Each dot represents the level of IgG (red) or IgM (blue) antibody for an individual. The SARS-CoV-2 antibody level expressed as the ratio is calculated by the chemiluminescence signal divides the cutoff value (S/CO). The dotted line means that the antibody level is at the cutoff value (S/CO=1).

Based on their age, all the subjects were further categorized into four subgroups: 0-18 years, 19-49 years, 50-64 years, and $>65$ years. The seroprevalence of antibodies to SARS-CoV-2 was higher in ocular surface disease with respect to age (Figure 5). A similar result was also observed when SARS-CoV-2 prevalence was compared among ocular surface diseases, no-ocular surface diseases and no-ocular diseases groups in each district (Figure 5). Similar results were also observed when SARS-
CoV-2 $\mathrm{IgM}^{+}$and $\mathrm{IgG}^{+}$prevalence was compared among ocular surface diseases, no-ocular surface diseases and noocular diseases groups with respect to age and district, respectively (Figure 5).

Survey seroprevalence was based on the following numbers of positive tests/sample sizes: 11/1211(May 2020), 27/2506 (June 2020), 14/1553 (July 2020), 15/1528 (August 2020), 13/1481 (September 2020), 14/1574 (October 2020), 15/1537

TABLE 3 | SARS-CoV-2 seroprevalence among different type of ocular surface diseases.

\begin{tabular}{|c|c|c|c|c|}
\hline & $\mathbf{n}$ & $\operatorname{lgG}^{+}$ & $\lg ^{+}$ & $\lg ^{+}$or $\lg G^{+}$ \\
\hline Ocular surface diseases & 1755 & 22 (1.25\%) & 25 (1.43\%) & $40(2.28 \%)$ \\
\hline Xerophthalmia & 174 & $4(2.30 \%)$ & $4(2.30 \%)$ & $6(3.45 \%)$ \\
\hline Keratitis & 231 & $3(1.30 \%)$ & $3(1.30 \%)$ & $4(1.73 \%)$ \\
\hline Conjunctival cyst & 80 & $0(0 \%)$ & $3(3.75 \%)$ & $3(3.75 \%)$ \\
\hline Conjunctivitis & 87 & $3(3.45 \%)$ & $3(3.45 \%)$ & $5(5.75 \%)$ \\
\hline Dacryocystitis & 117 & $1(0.85 \%)$ & $2(1.71 \%)$ & $3(2.56 \%)$ \\
\hline Conjunctival congestion & 97 & $0(0 \%)$ & $2(2.06 \%)$ & $2(2.06 \%)$ \\
\hline Trichiasis & 105 & $2(1.90 \%)$ & $0(0 \%)$ & $2(1.90 \%)$ \\
\hline Pterygium & 201 & $6(2.99 \%)$ & 4 (1.99\%) & 8 (3.98\%) \\
\hline Conjunctival nevi & 16 & $1(6.25 \%)$ & $0(0 \%)$ & $1(6.25 \%)$ \\
\hline Eyelid tumors & 304 & $2(0.66 \%)$ & $4(1.32 \%)$ & $6(1.97 \%)$ \\
\hline Others & 343 & $0(0 \%)$ & $0(0 \%)$ & $0(0 \%)$ \\
\hline
\end{tabular}




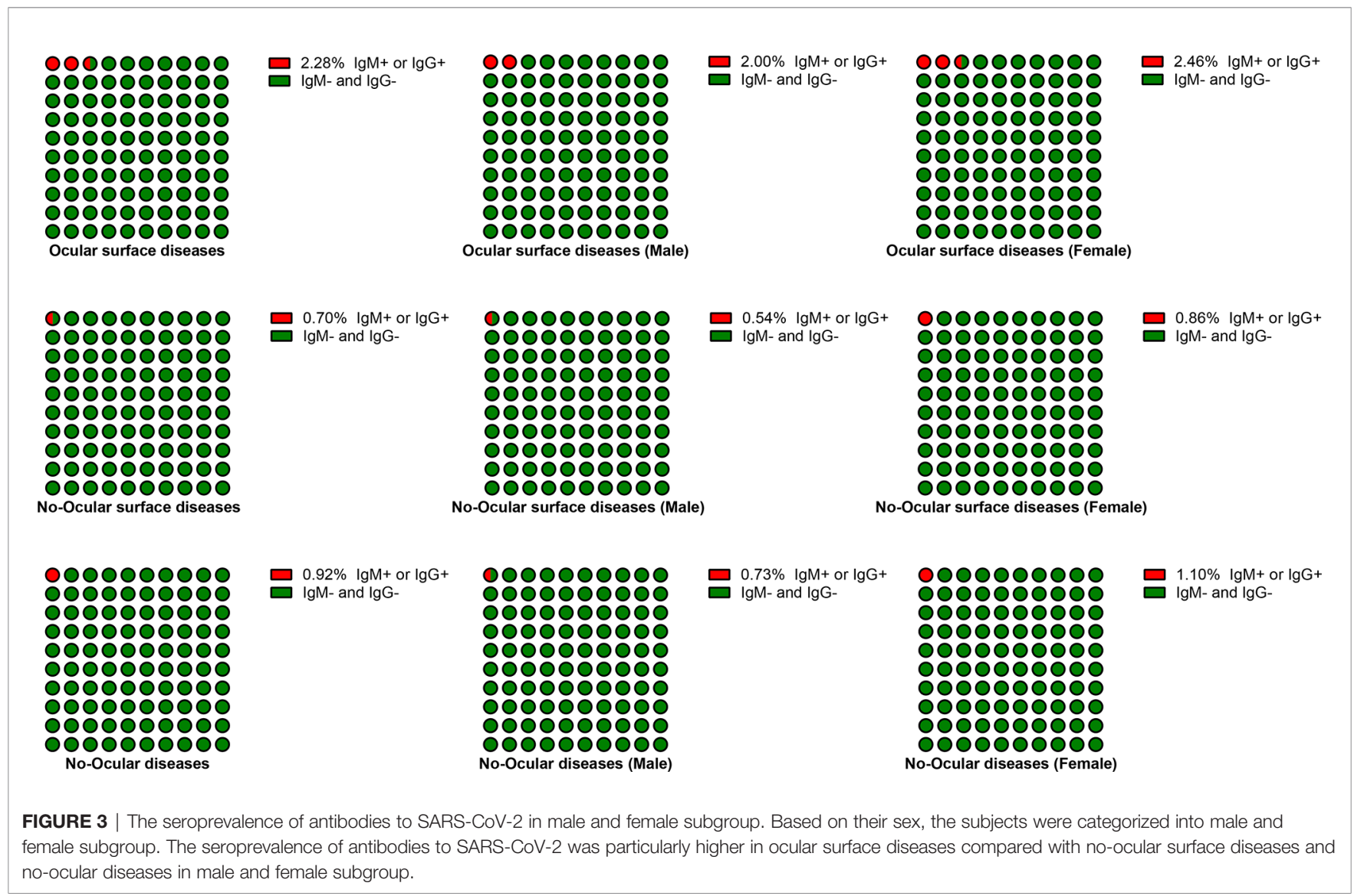

(November 2020), 15/1767 (December 2020), 13/1466 (January 2021), 28/3134 (February 2021), and 17/2400 (March 2021). In ocular surface disease population, greater than $1.4 \%$ of specimens had detectable SARS-CoV-2 antibodies at different times. However, in no-ocular surface disease and no-ocular disease population, less than $0.9 \%$ of specimens had detectable SARS-CoV-2 antibodies at different times (Figure 6A). Furthermore, similar results were also observed when SARS$\mathrm{CoV}_{-2} \mathrm{IgG}^{+}$(Figure 6B) and $\mathrm{IgM}^{+}$(Figure 6C) prevalence was compared among three groups at different times.

Logistic regression analyses were performed to identify the association between type of diseases and risk of susceptible to SARS-CoV-2 infection (Table 4). Logistic regression analyses revealed that ocular surface diseases [ocular surface diseases $v s$. no-ocular diseases $(\mathrm{p}=0.001, \mathrm{OR}=1.467,95 \% \mathrm{CI}=1.174-1.834)$; ocular surface diseases vs. no-ocular surface diseases $(\mathrm{p}<0.001$, $\mathrm{OR}=2.170,95 \% \mathrm{CI}=1.434-3.284$ ) ] were associated with increased risk of susceptible to SARS-CoV-2 infection, no matter $\mathrm{IgG}^{+}$ (Table 5) but also $\operatorname{IgM}^{+}$(Table 6).

\section{DISCUSSION}

Our results showed that the total positivity rate of IgM and IgG antibodies against SARS-CoV-2 (0.90\%) was close to the seropositivity rate $(0.8-1.68 \%)$ of community settings in several cities of China, as reported in other serological studies $(11,12)$. Although SARS-CoV-2 seroprevalence studies have previously been published (13-15), this is, to our knowledge, the first study to estimate the seroprevalence of antibodies against SARS-CoV-2 in patients with ocular diseases.

Remarkably, we found that the seroprevalence of antibodies to SARS-CoV-2 was particularly high in the patient population with ocular surface diseases. Clinical and scientific evidence suggests that the human ocular surface is susceptible to SARSCoV-2 infection $(16,17)$. A clinician wearing an N95 mask without eye protection was likely infected with SARS-CoV-2 through the eyes (16). An animal study showed that the virus can infect rhesus macaques via the conjunctival route (17). Zhou et al. (18) found that 8 of 121 COVID-19 patients studied had ocular symptoms. Wu et al. (2) reported that 12 of 38 COVID-19 patients had ocular manifestations consistent with conjunctivitis, including conjunctival hyperaemia, chemosis, epiphora, and increased secretions. Furthermore, Michael S Deiner et al. (19) also suggest a relationship between the COVID-19 pandemic and internet search patterns for some ocular surface conditions. In a word, it is obviously that there was a significantly association between COVID-19 and ocular surface conditions. However, it is still unknown ocular surface conditions were manifestation of COVID-19, or ocular surface conditions would be a risk factor for getting COVID-19? 


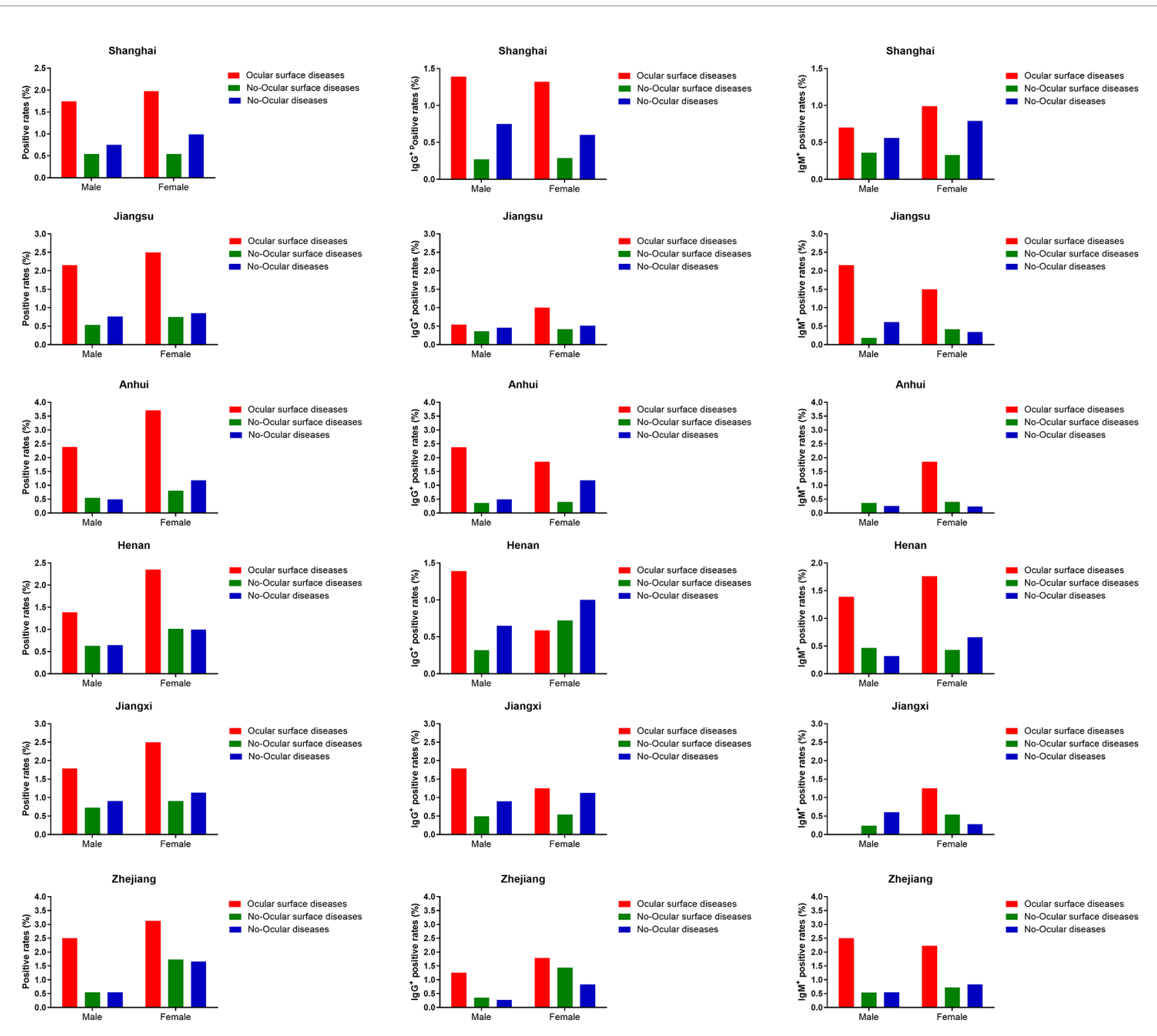

FIGURE 4 | The seroprevalence of antibodies to SARS-CoV-2 in districts subgroup. In each district, the seroprevalence of antibodies to SARS-CoV-2 was particularly higher in ocular surface diseases compared with no-ocular surface diseases and no-ocular diseases in male and female subgroup.

Recent studies have suggested that ACE2 and TMPRSS2 are expressed in human conjunctival and corneal cells $(5,20,21)$. ACE2 and TMPRSS2 is a major cellular-entry receptor for the SARS-CoV-2 virus, and higher ACE2 expression increases susceptibility to SARS-CoV-2. Collectively, clinical features of reported COVID-19 patients combined with our results indicate that COVID-19 is likely to be transmitted through the ocular surface. Thus, we speculate that ocular surface conditions would be a risk factor for getting COVID-19. Further prospective multicenter longitudinal studies are warranted to elucidate the underlying causal relationship. Furthermore, our previous study suggested that the SARS-CoV-2 receptor ACE2 is expressed in humans, especially in diseased conjunctival tissue (21). Therefore, in patients with ocular diseases-especially ocular surface diseases, such as keratitis and conjunctival cyst-ACE2 is possibly upregulated, making them more susceptible to SARS-CoV-2 infection (21). This may explain why seroprevalence was particularly high in patients with ocular surface diseases in this study.

Our study has certain limitations. First, the specimens were collected for clinical purposes from people seeking health care, potentially causing bias in the results. Second, owing to this study was a large-sample observational study, it is hard for us to obtain detailed demographic data such as diabetes, hypertension, and smoking which might result in bias, and it is hard for us to clarify the causal relationship between ocular surface diseases and SARS-CoV-2 infection. Therefore, multicentre prospective cohort studies are expected to clarify the causal relationship 

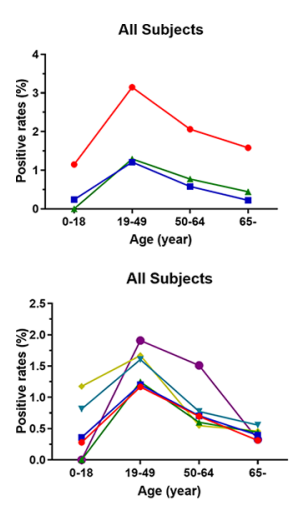

Shanghai

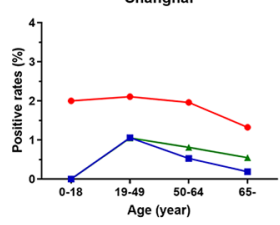

Jiangsu
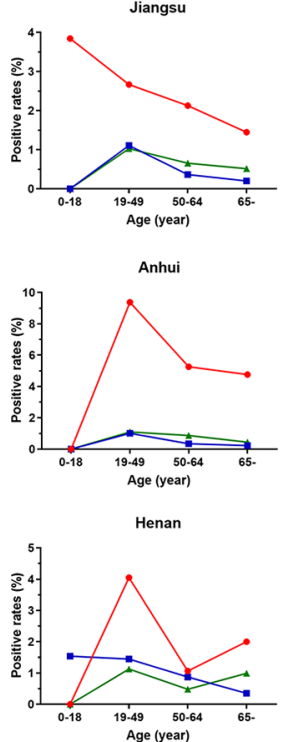

Jiangxi
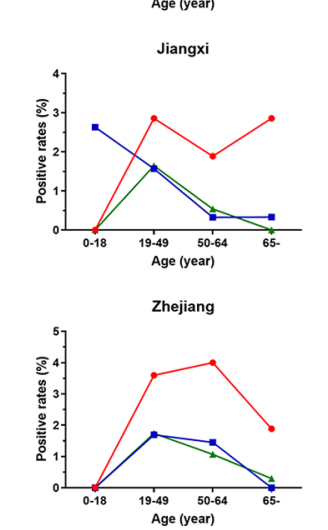

- Ocular surface diseases

- No-Ocular surface

- No-Ocular diseases

- Shanghai

- Jiangsu

* Anhui

$\rightarrow$ Henangxi

- Zhejiang

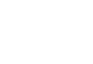

- Ocular surface diseases

- No-Ocular surface

- No-Ocular diseases

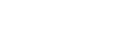

Ocular surface diseases

- No-Ocular surface

- No-Ocular diseases
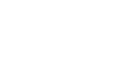

Ocular surface diseases

- No.Ocular surface

- No-Ocular diseases
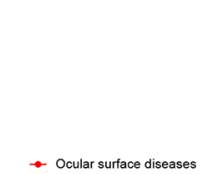

- No-Ocular surface

- No-Ocular diseases

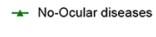

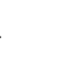
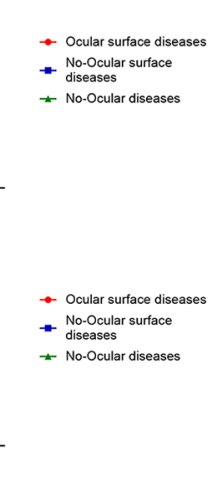

FIGURE 5 | The seroprevalence of antibodies to SARS-CoV-2 in age subgroup. Based on age, all the subjects were further categorized into four subgroups: 0-18 years, 19-49 years, 50-64 years, and >65 years. The seroprevalence of antibodies to SARS-CoV-2 was higher in ocular surface disease with respect to age. A similar result was also observed when SARS-CoV-2 prevalence was compared among ocular surface diseases, no-ocular surface diseases and no-ocular diseases groups in each district. 
A

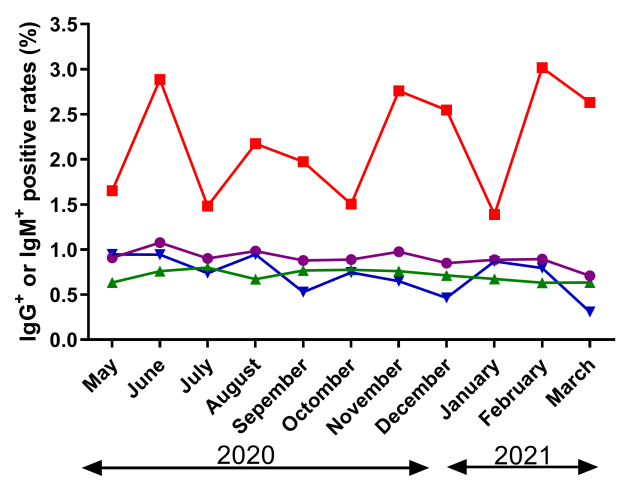

B

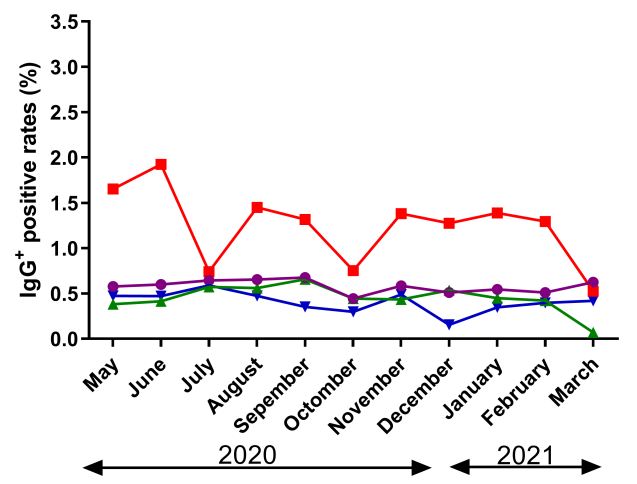

C

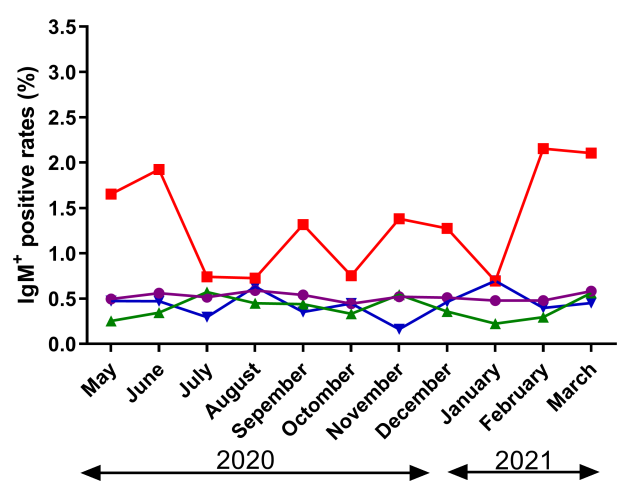

* No-Ocular diseases

- No-Ocular surface diseases

- Ocular surface diseases

$\rightarrow$ All subjects
* No-Ocular diseases

- No-Ocular surface diseases

- Ocular surface diseases

- All subjects

FIGURE 6 | The seroprevalence of antibodies to SARS-CoV-2 in time period subgroup $\left(\mathbf{A}: \operatorname{lgG}{ }^{+}\right.$or $\left.\operatorname{lgM} M^{+}, \mathbf{B}: \lg G^{+}, \mathbf{C}: \lg M^{+}\right)$. Survey seroprevalence was based on the following numbers of positive tests/sample sizes: 11/1211(May 2020), 27/2506 (June 2020), 14/1553 (July 2020), 15/1528 (August 2020), 13/1481 (September 2020), 14/1574 (October 2020), 15/1537 (November 2020), 15/1767 (December 2020), 13/1466 (January 2021), 28/3134 (February 2021), and 17/2400 (March 2021).

TABLE 4 | Associations between ocular surface diseases and risk of SARS-CoV-2 infection (lgG ${ }^{+}$or $\left.\operatorname{lgM}^{+}\right)$.

\begin{tabular}{|c|c|c|c|c|}
\hline & $\beta$ & $P$ value & OR & $95 \% \mathrm{Cl}$ \\
\hline Age & -0.005 & 0.485 & 0.995 & 0.983 to 1.008 \\
\hline Ocular surface diseases & 0.383 & 0.001 & 1.467 & 1.174 to 1.834 \\
\hline \multicolumn{5}{|l|}{ Model B } \\
\hline Age & -0.005 & 0.258 & 0.995 & 0.986 to 1.004 \\
\hline Gender & 0.298 & 0.131 & 1.347 & 0.915 to 1.982 \\
\hline
\end{tabular}

Model A: Ocular surface diseases vs. No-ocular diseases; Model B: Ocular surface diseases vs. No-ocular surfaces diseases. 
TABLE 5 | Associations between ocular surface diseases and risk of SARS-CoV-2 infection (lgG $\left.{ }^{+}\right)$.

\begin{tabular}{|c|c|c|c|c|}
\hline & $\beta$ & $P$ value & OR & $95 \% \mathrm{Cl}$ \\
\hline \multicolumn{5}{|l|}{ Model A } \\
\hline Age & -0.013 & 0.073 & 0.987 & 0.973 to 1.001 \\
\hline Ocular surface diseases & 0.333 & 0.011 & 1.395 & 1.081 to 1.800 \\
\hline \multicolumn{5}{|l|}{ Model B } \\
\hline Age & -0.007 & 0.158 & 0.993 & 0.983 to 1.003 \\
\hline Gender & -0.213 & 0.346 & 0.808 & 0.518 to 1.260 \\
\hline
\end{tabular}

Model A: Ocular surface diseases vs. No-ocular diseases; Model B: Ocular surface diseases vs. No-ocular surfaces diseases.

TABLE 6 | Associations between ocular surface diseases and risk of SARS-CoV-2 infection ((lgM+).

\begin{tabular}{|c|c|c|c|c|}
\hline & $\beta$ & $P$ value & OR & $95 \% \mathrm{Cl}$ \\
\hline Age & -0.010 & 0.178 & 0.990 & $0.975-1.005$ \\
\hline Ocular surface diseases & 0.520 & $<0.001$ & 1.682 & $1.297-2.182$ \\
\hline \multicolumn{5}{|l|}{ Model B } \\
\hline Age & -0.007 & 0.163 & 0.993 & $0.982-1.003$ \\
\hline Gender & -0.026 & 0.909 & 0.974 & $0.621-1.528$ \\
\hline
\end{tabular}

Model A: Ocular surface diseases vs. No-ocular diseases; Model B: Ocular surface diseases vs. No-ocular surfaces diseases.

between ocular surface diseases and SARS-CoV-2 infection. Last, the serological assays may have produced false negatives if samples were collected before infected individuals had a serological response.

In conclusion, the seroprevalence of SARS-CoV-2 was particularly high in population with ocular surface diseases, which suggested that there was a significant association between ocular surface disease and SARS-CoV-2 infection. Therefore, increasing awareness of eye protection during the pandemic is necessary, especially for individuals with ocular surface diseases.

\section{DATA AVAILABILITY STATEMENT}

The raw data supporting the conclusions of this article will be made available by the authors, without undue reservation.

\section{ETHICS STATEMENT}

The studies involving human participants were reviewed and approved by Eye and ENT Hospital of Fudan University. The patients/participants provided their written informed consent to participate in this study. Written informed consent was obtained from the individual(s) for the publication of any potentially identifiable images or data included in this article.

\section{AUTHOR CONTRIBUTIONS}

SL, XZ and WC designed this study. SL, YQ, LT, ZW, WC, XZ, and XS prepared and analysed the data. SL, WC, and XZ drafted the manuscript. All co-authors revised the manuscript together. All authors contributed to the article and approved the submitted version.

\section{FUNDING}

This work was supported by the Shanghai Municipal Commission of Health and Family Planning (20174Y0169 and 201840050), the State Key Program of National Natural Science Foundation of China (81430007), the subject of major projects of National Natural Science Foundation of China (81790641), the Shanghai Committee of Science and Technology of China (17410712500), and the top priority of clinical medicine center of Shanghai (2017ZZ01020) and Shanghai Science and Technology Committee Foundation grant (19411964600), National Natural Science Foundation of China (Grant No. 81770955), Joint research project of new frontier technology in municipal hospitals (SHDC12018103), Project of Shanghai Science and Technology (Grant No.20410710100), Clinical Research Plan of SHDC (SHDC2020CR1043B), Project of Shanghai Xuhui District Science and Technology (2020-015).

\section{SUPPLEMENTARY MATERIAL}

The Supplementary Material for this article can be found online at: https://www.frontiersin.org/articles/10.3389/fimmu.2021.695428/ full\#supplementary-material 


\section{REFERENCES}

1. Huang C, Wang Y, Li X, Ren L, Zhao J, Hu Y, et al. Clinical Features of Patients Infected With 2019 Novel Coronavirus in Wuhan, China. Lancet (2020) 395:497-506. doi: 10.1016/S0140-6736(20)30183-5

2. Wu P, Duan F, Luo C, Liu Q, Qu X, Liang L, et al. Characteristics of Ocular Findings of Patients With Coronavirus Disease 2019 (COVID-19) in Hubei Province, China. JAMA Ophthalmol (2020) 138:575-8. doi: 10.1001/ jamaophthalmol.2020.1291

3. Zhang X, Chen X, Chen L, Deng C, Zou X, Liu W, et al. The Evidence of SARS-CoV-2 Infection on Ocular Surface. Ocul Surf (2020) 18:360-2. doi: 10.1016/j.jtos.2020.03.010

4. Xie H-T, Jiang S-Y, Xu K-K, Liu X, Xu B, Wang L, et al. SARS-CoV-2 in the Ocular Surface of COVID-19 Patients. Eye Vis (Lond) (2020) 7:23. doi: 10.1186/s40662-020-00189-0

5. Collin J, Queen R, Zerti D, Dorgau B, Georgiou M, Djidrovski I, et al. CoExpression of SARS-CoV-2 Entry Genes in the Superficial Adult Human Conjunctival, Limbal and Corneal Epithelium Suggests an Additional Route of Entry via the Ocular Surface. Ocul Surf (2020) 19:190-200. doi: 10.1016/ j.jtos.2020.05.013

6. Gangaputra SS, Patel SN. Ocular Symptoms Among Nonhospitalized Patients Who Underwent COVID-19 Testing. Ophthalmology (2020) 127:1425-7. doi: 10.1016/j.ophtha.2020.06.037

7. Chen X, Chen Z, Azman AS, Deng K, Sun R, Zhao Z, et al. Serological Evidence of Human Infection With SARS-CoV-2: A Systematic Review and Meta-Analysis. Lancet Glob Health (2021) 9(5):e598-609. doi: 10.1016/S2214109X(21)00026-7

8. Vabret N, Britton GJ, Gruber C, Hegde S, Kim J, Kuksin M, et al. Immunology of COVID-19: Current State of the Science. Immunity (2020) 52:910-41. doi: 10.1016/j.immuni.2020.05.002

9. Zhao J, Yuan Q, Wang H, Liu W, Liao X, Su Y, et al. Antibody Responses to SARS-CoV-2 in Patients of Novel Coronavirus Disease 2019. Clin Infect Dis (2020) 71(16):2027-34. doi: 10.1093/cid/ciaa344

10. Gudbjartsson DF, Norddahl GL, Melsted P, Gunnarsdottir K, Holm H, Eythorsson E, et al. Humoral Immune Response to SARS-CoV-2 in Iceland. N Engl J Med (2020) 383(18):1724-34. doi: 10.1056/NEJMoa2026116

11. Xu X, Sun J, Nie S, Li H, Kong Y, Liang M, et al. Seroprevalence of Immunoglobulin $\mathrm{M}$ and $\mathrm{G}$ Antibodies Against SARS-CoV-2 in China. Nat Med (2020) 26:1193-5. doi: 10.1038/s41591-020-0949-6

12. Duan S, Zhou M, Zhang W, Shen J, Qi R, Qin X, et al. Seroprevalence and Asymptomatic Carrier Status of SARS-CoV-2 in Wuhan City and Other Places of China. PloS Negl Trop Dis (2021) 15:e0008975. doi: 10.1371/ journal.pntd.0008975

13. Murhekar MV, Bhatnagar T, Selvaraju S, Rade K, Saravanakumar V, Vivian Thangaraj JW, et al. Prevalence of SARS-CoV-2 Infection in India: Findings
From the National Serosurvey, May-June 2020. Indian J Med Res (2020) 152:48-60. doi: 10.4103/ijmr.IJMR_3290_20

14. Moscola J, Sembajwe G, Jarrett M, Farber B, Chang T, McGinn T, et al. Prevalence of SARS-CoV-2 Antibodies in Health Care Personnel in the New York City Area. JAMA (2020) 324:893-5. doi: 10.1001/jama.2020.14765

15. Havers FP, Reed C, Lim T, Montgomery JM, Klena JD, Hall AJ, et al. Seroprevalence of Antibodies to SARS-CoV-2 in 10 Sites in the United States, March 23-May 12, 2020. JAMA Intern Med (2020) 180(12):1576-86. doi: 10.1001/jamainternmed.2020.4130

16. Lu C-W, Liu X-F, Jia Z-F. 2019-Ncov Transmission Through the Ocular Surface Must Not Be Ignored. Lancet (2020) 395:e39. doi: 10.1016/S0140-6736 (20)30313-5

17. Deng W, Bao L, Gao H, Xiang Z, Qu Y, Song Z, et al. Ocular Conjunctival Inoculation of SARS-CoV-2 Can Cause Mild COVID-19 in Rhesus Macaques. Nat Commun (2020) 11:4400. doi: 10.1038/s41467-020-18149-6

18. Zhou Y, Duan C, Zeng Y, Tong Y, Nie Y, Yang Y, et al. Ocular Findings and Proportion With Conjunctival SARS-COV-2 in COVID-19 Patients. Ophthalmology (2020) 127:982-3. doi: 10.1016/j.ophtha.2020.04.028

19. Deiner MS, Seitzman GD, McLeod SD, Chodosh J, Hwang DH, Lietman TM, et al. Ocular Signs of COVID-19 Suggested by Internet Search Term Patterns Worldwide. Ophthalmology (2021) 128:167-9. doi: 10.1016/j.ophtha.2020.06.026

20. Zhou L, Xu Z, Castiglione GM, Soiberman US, Eberhart CG, Duh EJ. ACE2 and TMPRSS2 Are Expressed on the Human Ocular Surface, Suggesting Susceptibility to SARS-CoV-2 Infection. Ocul Surf (2020) 18(4):537-44. doi: $10.1016 /$ j.jtos.2020.06.007

21. Li S, Li D, Fang J, Liu Q, Cao W, Sun X, et al. SARS-CoV-2 Receptor ACE2 Is Expressed in Human Conjunctival Tissue, Especially in Diseased Conjunctival Tissue. Ocul Surf (2020) 19:249-51. doi: 10.1016/j.jtos.2020.09.010

Conflict of Interest: The authors declare that the research was conducted in the absence of any commercial or financial relationships that could be construed as a potential conflict of interest.

Publisher's Note: All claims expressed in this article are solely those of the authors and do not necessarily represent those of their affiliated organizations, or those of the publisher, the editors and the reviewers. Any product that may be evaluated in this article, or claim that may be made by its manufacturer, is not guaranteed or endorsed by the publisher.

Copyright $\odot 2021 \mathrm{Li}$, Qiu, Tang, Wang, Cao, Zhou and Sun. This is an open-access article distributed under the terms of the Creative Commons Attribution License (CC BY). The use, distribution or reproduction in other forums is permitted, provided the original author(s) and the copyright owner(s) are credited and that the original publication in this journal is cited, in accordance with accepted academic practice. No use, distribution or reproduction is permitted which does not comply with these terms. 